\title{
Random Amplified Polymorphic Dna-PCR Typing of Vibrio parahaemolyticus Isolated from Local Cockles (Anadara granosa)
}

\author{
${ }^{1}$ Lesley Maurice Bilung, ${ }^{2}$ Son Radu, ${ }^{3}$ Abdul Rani Bahaman, ${ }^{4}$ Raha Abdul Rahim, ${ }^{4}$ Suhaimi Napis \\ ${ }^{2}$ Cheah Yoke Kqueen, ${ }^{2}$ Chandrika Murugaiah, ${ }^{4}$ Yousr Abdul Hadi, ${ }^{2}$ Tunung Robin and \\ ${ }^{5}$ Mitsuaki Nishibuchi \\ ${ }^{1}$ Department of Biotechnology, Faculty of Science and Engineering \\ University Tunku Abdul Rahman, Genting-Klang Road, 53300 Setapak, Kuala Lumpur, Malaysia \\ ${ }^{2}$ Department of Food Science, Faculty of Food Science and Technology \\ University Putra Malaysia, 43400 UPM, Serdang, Selangor, Malaysia \\ ${ }^{3}$ Faculty of Veterinary Medicine, University Putra Malaysia, 43400 UPM, Serdang, Selangor, Malaysia \\ ${ }^{4}$ Department of Cell and Molecular Biology, Faculty of Biotechnology and Biomolecular Sciences \\ University Putra Malaysia, 43400 UPM, Serdang, Selangor, Malaysia \\ ${ }^{5}$ Center for Southeast Asian Studies, Kyoto University, Yoshida, Sakyo-ku, Kyoto, 606-8501, Japan
}

\begin{abstract}
Randomly amplified polymorphic DNA (RAPD) was used in this study to examine the genetic relatedness among the Vibrio parahaemolyticus strains. In the analysis by RAPD-PCR, the size for RAPD fragments ranged from 0.25 to $10.0 \mathrm{~kb}$ with average number of ten bands. The RAPD profiles revealed a high level of DNA sequence diversity within the Vibrio parahaemolyticus strains tested. Hence, this study, demonstrated that the local cockles (Anadara granosa) in the study area are populated by genetically polymorphic strains of $V$. parahaemolyticus. In addition, RAPD-PCR is simple, robust and sensitive typing methods to differentiate the $V$. parahaemolyticus strains.
\end{abstract}

Key words: RAPD-PCR, cockles, Vibrio parahaemolyticus

\section{INTRODUCTION}

Vibrio parahaemolyticus is a Gram-negative marine bacterium that can cause seafood-borne gastroenteritis and traveler's diarrhea in humans after they consumed contaminated raw or partially cooked fish or shellfish, particularly oysters ${ }^{[1]}$ or exposure to a marine environment. The organism is widely disseminated in estuarine environments throughout the world. Outbreaks of $V$. parahaemolyticus food poisoning are most common in Japan and Southeast Asia, although they occurred occasionally in other parts of the world ${ }^{[2]}$, and are associated with diverse serovars. Recent studies ${ }^{[3,4]}$ have shown the emergence of O3:K6 that appears to have the potential to spread and to be associated with infections more than other serovars.

Epidemiological typing of pathogens such as Listeria monocytogenes, V. parahaemolyticus and Salmonella enteriditis has become more and more important in public health control. Molecular typing procedures are applied to show clonal and close relationship between isolates of one species. Thus, it is possible to identify pathogen reservoirs and to follow up the regional and global spread of pathogens. Some typing methods also give an insight into the evolutionary dynamics of the bacterial genome. The classical and microbiological methods, based on the identification of phenotypic markers, while perfectly adequate to identify microorganisms on the level of species, are often not reliable enough when it comes to differentiating further into individual strains. This is where DNA-based procedures like Polymerase Chain Reaction (PCR) or DNA-sequencing approaches helped epidemiological investigations to be conducted more rapidly and thoroughly ${ }^{[5]}$. RAPD analysis is a commonly used method in PCR. This fingerprint has been used for typing and differentiation of bacteria and, increasingly, for the study of genetic relationships between strains and species of microorganisms, plants and animals ${ }^{[6]}$. Nowadays, molecular typing methods are necessary for proving the similarity between the isolates. The aim of the present investigation was to use RAPD-PCR assay to generate polymorphism in DNA patterns amenable to the differentiation of the $V$. parahaemolyticus strains isolated from cockles.

\section{MATERIALS AND METHODS}

Bacterial strains: The sixty-two isolates of $V$. parahaemolyticus isolated from local cockles obtained from a harvesting site in Tanjong Karang, Kuala Selangor were examined. These strains were confirmed by using specific-PCR targeting the species-specific ToxR region in $V$. parahaemolyticus as mentioned by Kim et al. ${ }^{[7]}$ and Dileep et al. ${ }^{[8]}$.

Corresponding Author: Lesley Maurice Bilung, Department of Biotechnology, Faculty of Science and Engineering, University Tunku Abdul Rahman, Genting-Klang Road, 53300 Setapak, Kuala Lumpur, Malaysia 
Genomic DNA isolation: Prior to amplification, genomic DNA of the $V$. parahaemolyticus strains were extracted by mini-preparation method of Ausubel et al. ${ }^{[9]}$.

\section{Randomly amplified polymorphic DNA (RAPD) RAPD-PCR amplification: Amplification reactions} were performed in $25 \mu \mathrm{l}$ volume containing $2.5 \mathrm{mM}$ $\mathrm{MgCl}_{2}, 200 \mu \mathrm{M}$ each dATP, dCTP, dGTP and dTTP (Promega), $0.5 \mu \mathrm{M}$ primer, 1.25 Units of Taq polymerase, 10-20 ng of genomic DNA. Amplifications were carried out in the thermal cycler (Perkin Elmer Cetus 2400) for 35 cycles of $1 \mathrm{~min}$ at $94^{\circ} \mathrm{C}, 1 \mathrm{~min}$ at $36^{\circ} \mathrm{C}$ and $2 \mathrm{~min}$ at $72^{\circ} \mathrm{C}$. A final elongation step at $72^{\circ} \mathrm{C}$ for 5 min was included. Amplification products were fractionated by electrophoresis through $1.5 \%$ agarose gel and detected by staining with ethidium bromide. DNA ladder (Promega) was used as DNA size marker.

Primers: Ten random primers having the $50 \% \mathrm{G}+\mathrm{C}$ contents gene sequence (10-mer) were screened and three primers, the Gen 1-50-03 (5'-CTT GAG TGG A3'), Gen 1-50-04 (5'-TCC TCAAGA C-3') and Gen 150-08 (5'-GAG ATG ACG A-3') were selected for further study as they provide reproducible and discriminatory patterns.

\section{RESULTS}

Of the ten random primers, Gen 1-50-03, Gen 150-04 and Gen 1-50-08 were chosen for differentiation of $V$. parahaemolyticus isolates. The representative RAPD fingerprinting profiles obtained were displayed in Fig. 1-3. The size for RAPD fragments ranged from 0.25 to $10.0 \mathrm{~kb}$ with average number of ten bands for each primer used. All results from RAPD-PCR were analyzed with the GelCompare software (version 4.1). The overall achievable patterns were used to construct dendrograms using the UPGMA (unweighted pair group average method) clustering algorithms.

\section{DISCUSSION}

The application of PCR-based techniques has had a revolutionary impact on the diagnosis of infectious disease. A rapid typing method based on the random amplification of polymorphic DNA segments (RAPDPCR) has been described ${ }^{[10,11]}$. This method has been successfully used in the characterization of several organisms including $V$. parahaemolyticus and other vibrios $^{[1,12-14]}$. In this study, a library of 10 primers $(50 \% \mathrm{G}+\mathrm{C})$ was examined for suitability. Of these 10 primers, three (GEN1-50-03, GEN1-50-08 and GEN150-08) proved to be useful primers for the RAPD analysis of strains of $V$. parahaemolyticus. In order to obtain a sufficient number of polymorphic bands that will permit reliable comparison, the three different 10mer primers were used in amplification reactions. Previously, Shangkuan et al. ${ }^{[12]}$ demonstrated the importance of using a minimum of three different primers to discriminate different isolates of toxigenic $V$. cholerae. The results of using three primers in this study indicate that RAPD provides a high degree of discrimination between $V$. parahaemolyticus strains, with amplified products ranging from 0.25 to $10.0 \mathrm{~kb}$. To determine the reproducibility of the RAPD analysis procedure, RAPD-PCR was repeated at least three times for DNA from each strain, so that the amplification profiles for each attempt could be compared. We found that profiles for a given strain to be highly reproducible, with very little variation from one RAPD analysis to another.

Our results show that all strains of $V$. parahaemolyticus isolates generated appropriate fragments. Averagely ten amplified DNA bands were resolved by agarose gel electrophoresis in these isolates. Using primer GEN1-50-03, V. parahaemolyticus presented bands with common sizes in the range of 1000 bp - 1500 bp (VP1-VP16, VP18 - VP21, VP23 VP38) and $1500 \mathrm{bp}$ - 2000 bp (VP39 - VP62). The isolates examined gave bands with molecular sizes of 500 bp - 750 bp (VP1 - VP17, VP43 - VP62), 750 bp - 1000 bp (VP18 - VP27, VP29 - VP62), 1000 bp (VP1 - VP17), 1000 bp - 1500 bp (VP33 - VP42) and 1500 bp - 2000 bp (VP1 - VP32, VP43 - VP62) using primer GEN1-50-04. Distinct band at the range size of $2000 \mathrm{bp}-2500 \mathrm{bp}$ were detected for six $V$. parahaemolyticus isolates (VP4, VP8, VP12, VP21, VP24 and VP26). Dominant bands of the molecular size in the range of $750 \mathrm{bp}-1000 \mathrm{bp}$ (VP1 - VP18) and $1000 \mathrm{bp}$ - $1500 \mathrm{bp}$ (VP1 - VP62) were observed using primer GEN1-50-08. However, two isolates (VP17 and VP22) and one isolate (VP28) was not typeable using primer GEN1-50-03 and GEN1-50-04, respectively, but all strains were able to be amplified using primer GEN1-50-08.

RAPD-PCR was used as a mean to determine the clonal relatedness of the isolates in this study by their chromosomal polymorphism. The data from the primer GEN1-50-03, GEN1-50-04 and GEN1-50-08 was used to generate a distance matrix. By using Gel Compar version 4.1 software, typable $V$. parahaemolyticus isolates were placed in 7,6 and 10 clusters for primer GEN1-50-03, GEN1-50-04 and GEN1-50-08, respectively (Fig. 1-3). At $24 \%$ and $10 \%$ similarity, the isolates were classified into 2 major clusters (R1 and R2) and one major cluster (R3) with a single isolate (VP59) using primer GEN1-50-03 and GEN1-50-04, respectively. These main clusters labeled as R1, R2 and R3 were sub-divided into 6, 5 and 9 subclusters, respectively. Our RAPD analysis revealed 

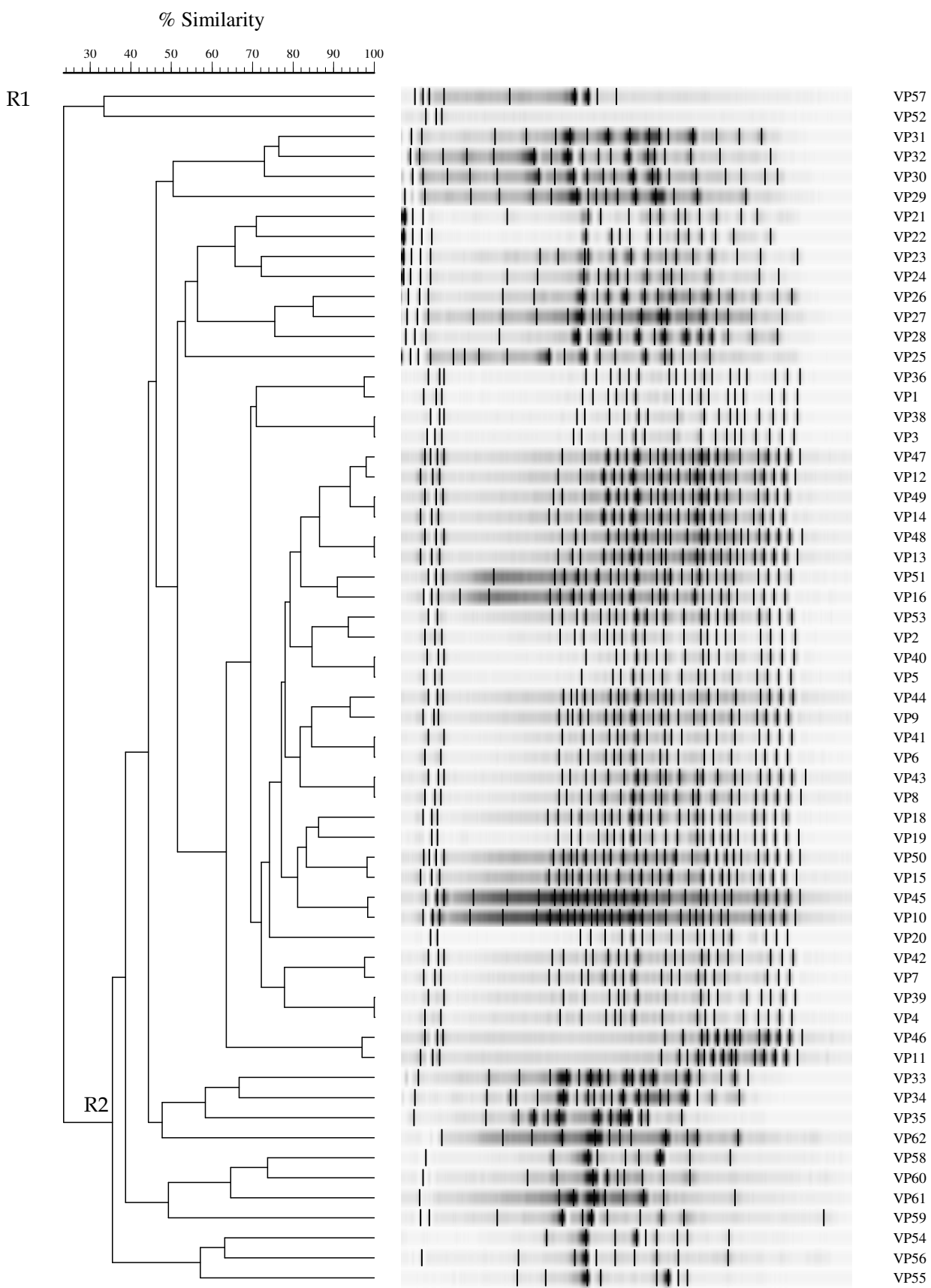

Fig. 1: Dendrogram showing RAPD profiles of typeable $V$. parahaemoyticus isolates using GEN1-50-03

heterogeneous isolates of $V$. parahaemolyticus in cockles by using these two primers (GEN1-50-03 and GEN1-50-04). On the other hand, the isolates were observed as homogeneous when typed using primer GEN1-50-08, as the cluster analysis shows that at $52 \%$ similarity the isolates were clustered into 1 major cluster and a single isolate (VP25). Our results are supported by others who observed homogeneous or heterogeneous isolates of $V$. parahaemolyticus or Vibrio spp. in cockles or shellfish by RAPD-PCR assays ${ }^{[15-18]}$. The high diversity among the RAPD-types probably means that contamination of cockles by $V$. parahaemolyticus is derived from wide spectra sources of contamination. It is also likely that different strains of $V$. parahaemolyticus predominate in cockles in the study area.

The results obtained by RAPD-PCR indicated that multiple clones of $V$. parahaemolyticus were present in the environment. These observations are in agreement with those of others who demonstrated heterogeneity 

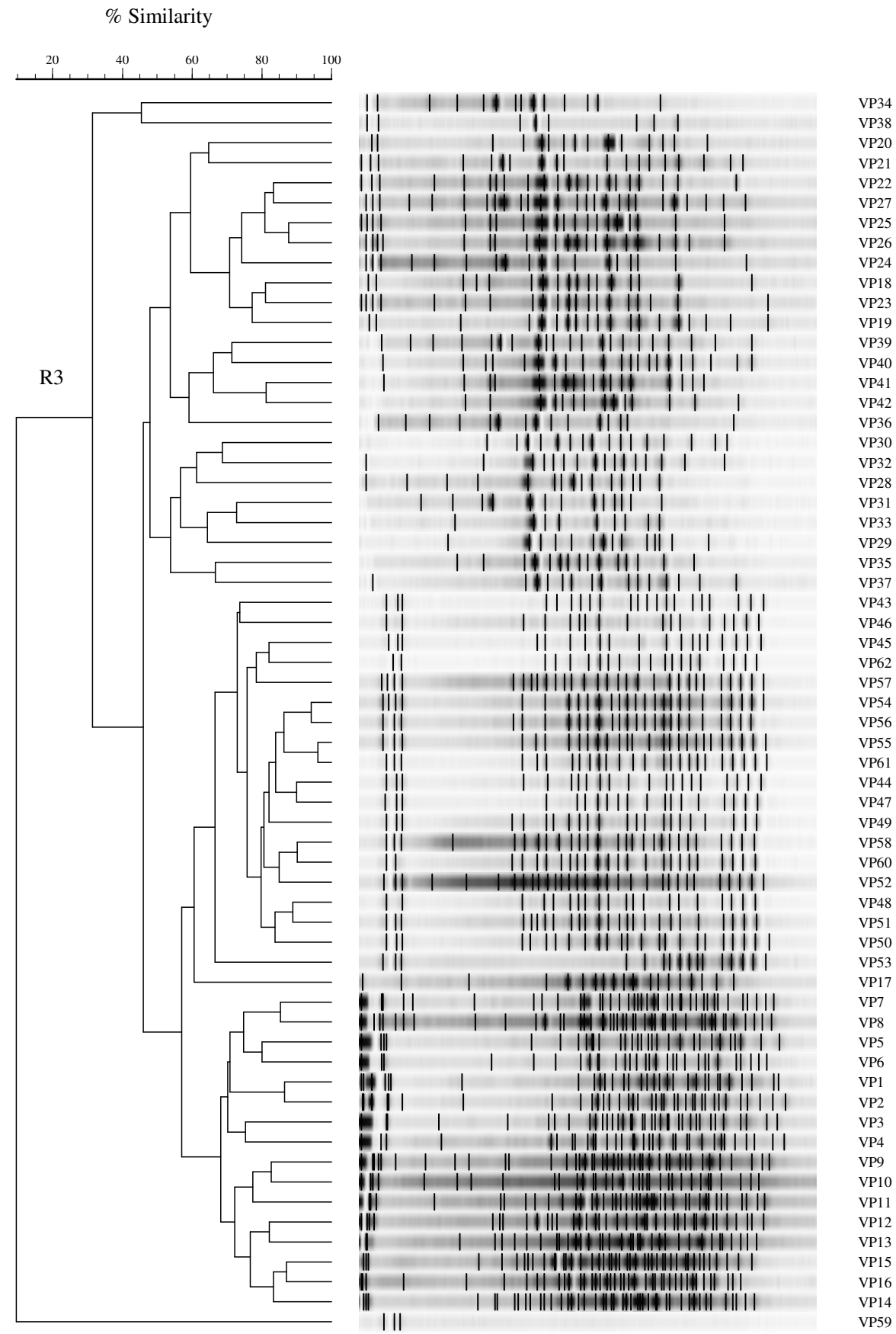

Fig. 2: Dendrogram showing RAPD profiles of typeable $V$. parahaemoyticus isolates using GEN1-50-04

in V. parahaemolyticus or Vibrio spp. ${ }^{[15,19]}$. Many researches reported the abundance of $V$. parahaemolyticus during summer in temperate zone when temperature was above $25^{\circ} \mathrm{C}^{[1,20,21]}$, whereas the organism is expected to be prevalent throughout the year in the tropical zone like Malaysia ${ }^{[22]}$. The hot climate in Malaysia and other tropical countries make conditions more favourable for the growth of $V$. parahaemolyticus, increasing the risk of outbreaks associated with this organism.

In summary, results from this study demonstrated that genotyping $V$. parahaemolyticus isolates by using RAPD -PCR is feasible for differentiation of various strains. RAPD-PCR has shown to be rapid, sensitive, discriminative and cost effective in typing the $V$. parahaemolyticus isolates from cockles caught in Tanjong Karang, Kuala Selangor. 


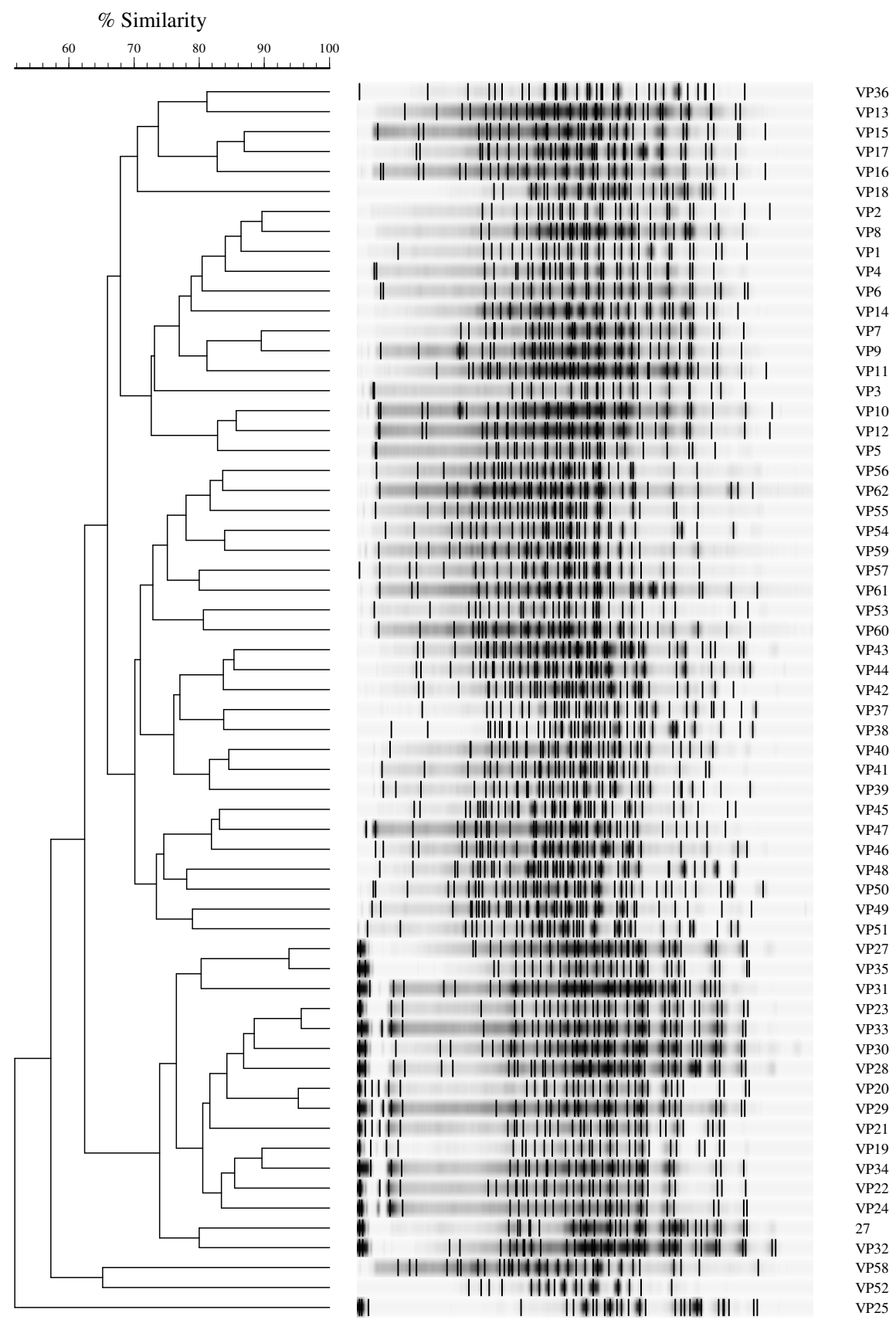

Fig. 3: Dendrogram showing RAPD profiles of typeable $V$. parahaemoyticus isolates using GEN1-50-08

\section{ACKNOWLEDGEMENTS}

Funding for this research was made possible by the IRPA grant from Malaysian Government.

\section{REFERENCES}

1. Khan, A.A., S. McCarthy, R.F. Wang and C.E. Cerniglia, 2002. Characterization of United States outbreak isolates of Vibrio parahaemolyticus using enterobacterial repetitive intergenic consensus (ERIC) PCR and development of a rapid PCR method for detection of O3:K6 isolates. FEMS Microbiol. Lett., 206: 209-214.
2. Nishibuchi, M., 2004. Vibrio parahaemolyticus. In International Handbook of Foodborne Pathogens. Ed. M.D. Milliots and J.W. Bier, pp: 237-252. United States: Marcel Dekker, Inc.

3. Wong, H.C., S.H. Liu, T.K. Wang, C.L. Lee, C.S. Chiou, D.P. Liu, M. Nishibuchi and B.K. Lee, 2000. Characterization of Vibrio parahaemolyticus O3:K6 from Asia. Appl. Environ. Microbiol., 66: 3981-3986.

4. Laohaprertthisan, V., A. Chowdhury, U. Kongmuang, S. Kalnauwakul, M. Ishibashi, C. Matsumoto and M. Nishibuchi, 2003. Prevalence and serodiversity of the pandemic clone among the clinical strains of Vibrio parahaemolyticus isolated in southern Thailand. Epidemiol. Infect., 130: 395-406. 
5. Woodford, N. and A.P. Johnson, 1998. Molecular bacteriology. United States: Humana Press.

6. Oakey, H.J., L.F. Gibson and A.M. George, 1998. Co-migration of RAPD-PCR amplicons from Aeromonas hydrophila. FEMS Microbiol. Lett., 164: 35-38.

7. Kim, Y.B., J. Okuda, C. Matsumoto, N. Takahashi, S. Hashimoto and M. Nishibuchi, 1999. Identification of Vibrio parahaemolyticus strains at the species level by PCR targeted to the toxR gene. J. Clin. Microbiol., 37: 1173-1177.

8. Dileep, V., H.S. Kumar, Y. Kumar, M. Nishibuchi, I. Karunasagar and I. Karunasagar, 2003. Application of polymerase chain reaction for detection of Vibrio parahaemolyticus associated with tropical seafoods and coastal environment. Lett. Appl. Microbiol., 36: 423-427.

9. Ausubel, F.M., R. Brent, R.E. Kingston, D.D. Moore, J. Sideman, J. Smith and K. Struhl, 1987. Current Protocol in Molecular Biology. New York: Wiley.

10. Welsh, J. and M. McClelland, 1990. Fingerprinting genome using PCR with arbitrary primers. Nucleic Acid Residues, 18: 7213-7218.

11. Williams, J.G.K., A.R. Kubelik, K.J. Livak, J.A. Rafalski and S.V. Tingey, 1990. DNA polymorphisms amplified by arbitrary primers are useful as generic markers. Nucleic Acid Residues, 18: 6531-6535.

12. Shangkuan, Y.H., C.M. Tsao and H.C. Lin, 1997. Comparison of Vibrio cholerae $\mathrm{O} 1$ isolates by polymerase chain reaction fingerprinting and ribotyping. J. Med. Microbiol., 46: 941-948.

13. Warner, J.M. and J.D. Oliver, 1999. Randomly Amplified Polymorphic DNA analysis of starved and viable but nonculturable Vibrio vulnificus cells. Appl. Environ. Microbiol., 64: 3025-3028.

14. Wong, H.C. and Lin, C.H. 2001. Evaluation of typing of Vibrio parahaemolyticus by three PCR methods using specific primers. J. Clin. Microbiol., 39: 4233-4240.
15. Son, R., E.H. Nasreldin, H. Zaiton, L. Samuel, G. Rusul and F. Nimita, 1998. Characterisation of Vibrio vulnificus isolated from cockles (Anadara granosa): antimicrobial resistance, plasmid profiles and random amplification of polymorphic DNA analysis. FEMS Microbiol. Lett., 165: 139-143.

16. Micky, V., 2001. Molecular characterization of Vibrio cholerae $\mathrm{O} 1$ from outbreak cases in Miri, Sarawak. Master Thesis. Universiti Putra Malaysia.

17. Nasreldin, E.H., 2001. Prevalence of pathogenic Vibrio spp., growth survival and molecular characterisation of Vibrio cholera serotype O1, O139 and non-O1/O139 isolated from seafood in Malaysia. Ph.D Thesis. Universiti Putra Malaysia, Malaysia.

18. Gwendelynne, B.T., 2004. Molecular characterization of Vibrio parahaemolyticus isolated from coastal seawater in Peninsular Malaysia. Master Thesis. Universiti Putra Malaysia, Malaysia.

19. Bhanumathi et al., 2003. Molecular characterization of Vibrio cholera O139 Bengal isolated from water and the aquatic plant Eichhornia crassipes in the River Ganga, Varanasi, India. Appl. Environ. Microbiol., 69: 2389-2394.

20. Centers for Disease Control and Prevention (CDC), 1998. Outbreak of Vibrio parahaemolyticus infections associated with eating raw oysters Pacific Northwest, 1997. Morbidity Mortality Weekly Report, 47: 457-462.

21. Centers for Disease Control and Prevention, (CDC), 1999. Outbreak of Vibrio parahaemolyticus infections associated with eating raw oysters and clams harvested from Long Island SoundConnecticut, New Jersey and New York, 1998. Morbidity Mortality Weekly Report, 48: 48-51.

22. Elhadi, N., S. Radu, C.H. Chen and M. Nishibuchi, 2004. Prevalence of potentially pathogenic Vibrio species in the seafood marketed in Malaysia. J. Food Protection, 67: 1469-1475. 